\title{
Design of a Hydraulic Robot Shoulder Based on a Combinatorial Mechanism
}

\author{
Vincent Hayward \\ Center for Intelligent Machines, McGill University \\ 3480 University Street \\ Montréal, Québec, Canada, H3A 2A7
}

\begin{abstract}
In previous papers, I have argued that while parallel mechanisms are well known for their favorable structural properties, their utility is generally limited by an inherently small workspace. I have also argued that proper use of actuator redundancy can simultaneously increase the workspace, remove singularities, and dramatically improve overall kinematic, structural, and actuator performance, while keeping the complexity low. This paper discusses a prototype shoulder joint more appropriately described as a combinatorial mechanism which exhibits the features. Additional benefits in terms of modularity, self-calibration, reliability, self-test, and degraded modes of operation are briefly discussed in the conclusion.
\end{abstract}

\section{Introduction}

While a great deal pf research activity is devoted to the design of robot manipulator wrists (see [18]) and articulated hands, the design of other joints or groups of joints is not less challenging. A robot manipulator, like most technological creations: automobiles, airplanes, excavators, etc., is an integrated machine. Weakness of any of its constituents: actuators, sensors, structural properties, materials, kinematic properties, dynamic properties, control techniques, and many more factors, will affect dramatically the performance of the complete machine [5].

Note that the great majority of existing manipulator arms, man-made or found in Nature, can be viewed as the assembly of three joint groups: the shoulder group which provides for the overall orientation or the pointing direction of the manipulator, the elbow group which provides for radial extension or reach, and the wrist group which provides for the final orientation of the effector. Of course, there are exceptions to this rule: some crustacean or insect limbs, for example, are not so clearly organized. This rule nevertheless appears to be well enforced for all larger animals.

Among man-made manipulators in use in the manufacturing industry (welding, painting, assembly, part handling, deburring, polishing, etc.), the author has seldom seen an exception to this rule. In the application of robotics for intervention in remote or hazardous locations, this rule seems to be followed even more meticulously: for undersea, outerspace, and other applications, among many existing or proposed designs that the author has surveyed, the only ex- 
In Experimental Robotics 3, Yoshikaswa, T., Myiazaki, F (Eds.),

Lecture Notes in Control and Information Sciences 200. Springer Verlag. pp. 297--309.

\section{8}

ception found are the "variable truss manipulators" dealt with in the next paragraph.

In research and development laboratories, there are numerous exceptions to the suggested rule. Although it would be lengthy to discuss them all, it may be noticed that the greatest majority of these exceptions either fall in the category of trunks, spines, snakes, tentacles, and other segmented designs (for example see [3]), or in the category of platform manipulators (see [17] for example). These categories often overlap (see [15] and [4] for example).

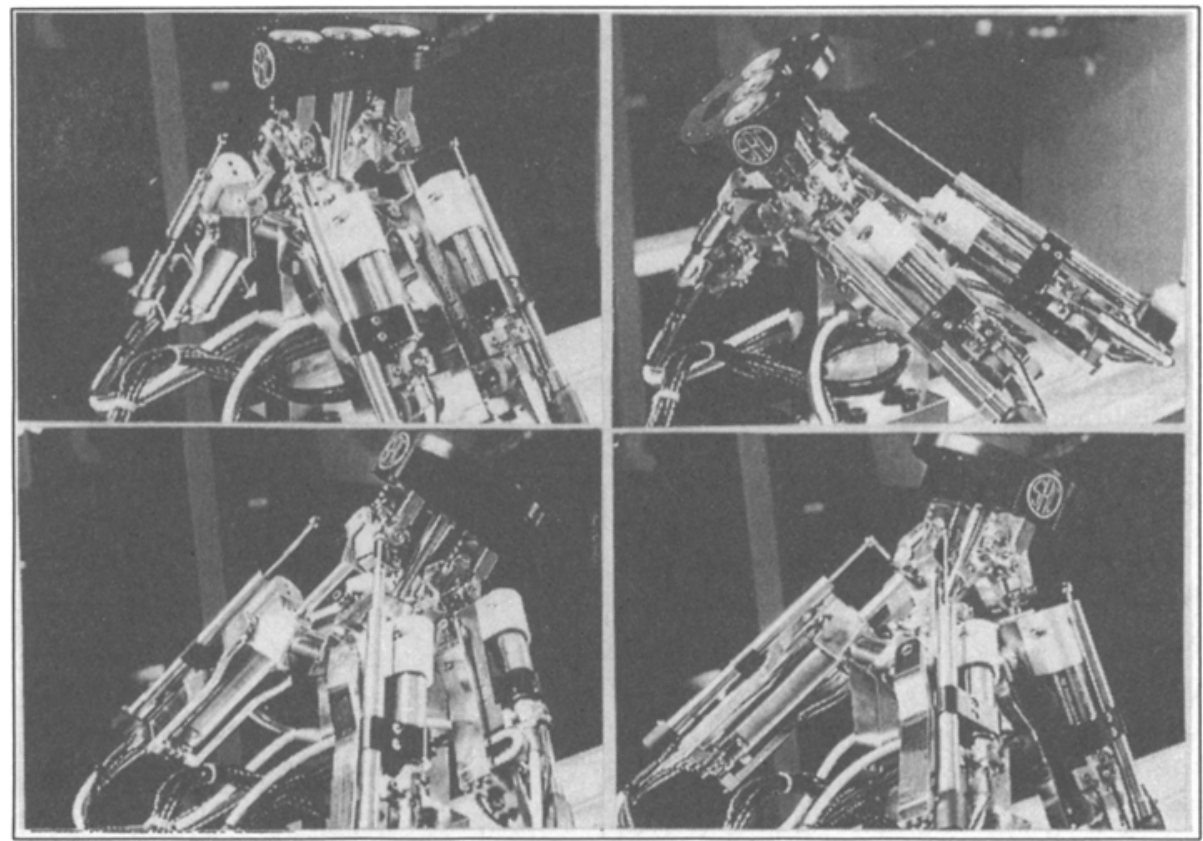

Figure 1.

Following what has just been said, some liberties with the conventional terminology are taken: the word 'joint' will refer to a group of joints, actuated or passive, instrumented or not, which contribute toward a common function: orientation or extension, as in the 'shoulder joint' or the 'wrist joint'. In this very brief survey, a large number of examples could not be commented on. Since the focus is on hydraulic actuation, the reader is referred to three recent designs of hydraulic manipulators $[12,19,21]$ for purposes of comparison. All three examples have a serial linkage architecture, although in the first two cases, larger versions of these manipulators have one or two proximal revolute joints replaced by four-bar 'crank-piston' joints.

Here, the design of a shoulder joint of unconventional architecture is described and the characteristics of a prototype discussed. This paper is a sequel to [7]. Please see Fig. 1 for a view of the prototype. 


\section{Design}

The major characteristic of a manipulator shoulder joint is that it works with the worst possible mechanical advantage, and yet its bulk and weight must be minimized, although this last requirement is obviously less critical than it is for distal joints. In the applications of robots in hazardous environments, this requirement is nevertheless crucial because a manipulator is typically not a grounded structure, but instead, is supported by a gross positioning device: a vehicle, a boom, or a crane. ${ }^{1}$ In [7], a promising architecture to achieve light weight, high mobility and favorable structural characteristics is discussed and represented in Fig. 2.
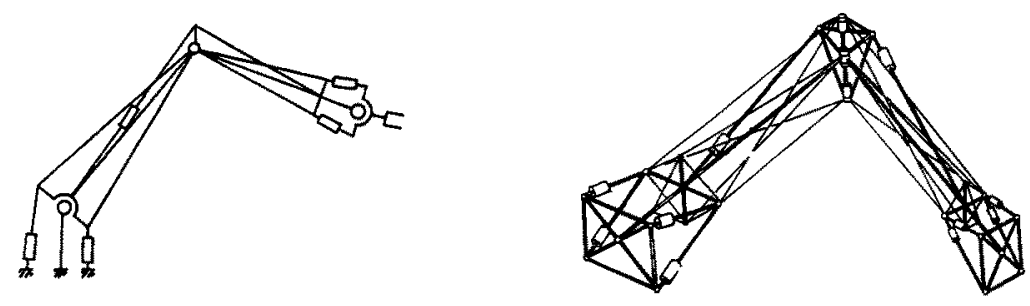

Figure 2. On the right, a spatial version of the planar case shown on the right is diagrammed.

The kinematic/structural concept is shown on Fig 3. According to the prevailing terminology, such a mechanism is termed actuator redundant. The author much prefers the term combinatorial because this adjective describes more accurately the underlying concept. No actuator is 'redundant': all are used and well. Their combined utility can easily be appreciated by observing the dramatic loss of performance of the entire system when one actuator in removed (much more than $25 \%$, anyway we look at it). Interestingly, the loss of an actuator does not leave the device completely crippled, in fact, a notable level of functionality is preserved, albeit in a greatly reduced controlled workspace (by about an order of magnitude).

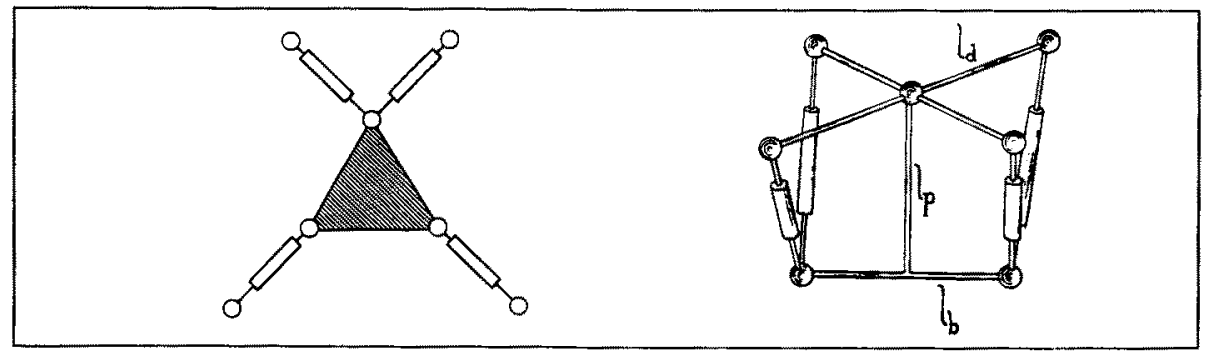

Figure 3. Combinatorial spherical mechanism, analogous to the planar version shown on the left

\footnotetext{
${ }^{1}$ This paragraph applies to human arms as well!
} 


\subsection{Kinematic Concept}

The operation of this architecture is best understood by viewing it as a combination of four piston-crank systems working cooperatively in a differential fashion, a spatial version of a two piston-one-crank mechanism, so to speak. In this case, four topological regions can readily be distinguished, labelled by the relative velocities of the two pistons, as seen on the Fig. 4.

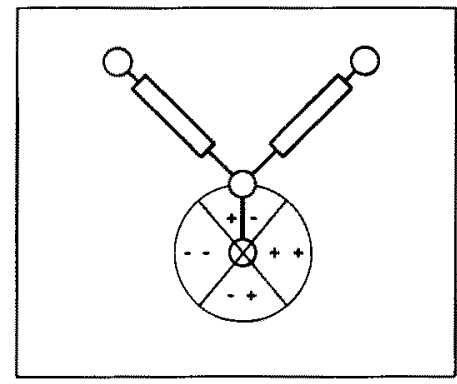

Figure 4. The four topological regions of the simplest combinatorial mechanism.

For the more general case discussed in this paper, in the vicinity of the central position, tilting motions occur when the pistons attached to opposite edges of the square platform move with velocities whose signs are different, while swiveling motions occur when pistons in opposite corners have velocities with differing signs as illustrated in Fig. 5.
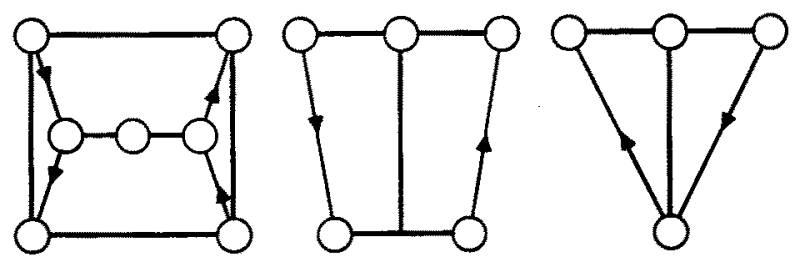

Figure 5. Velocity sign combinations.

Motions with all four equals velocity signs are kinematically prevented since the platform is constrained to a spherical motion. These sign relationships are captured by the signs of each entry in the Jacobian matrix of the mechanism's kinematic map [8]. Thus, there is an upper bound of $2^{12}$ of such topological regions, but at this point there is no proof that they all exist. The transition from one region to another is illustrated in the Fig. 6 during a swiveling motion with no tilt.

\subsection{Expected Performance}

The study in [13] indicates that the theoretical optimal workspace free of singularities (all parts with zero thickness) is as large as $180^{\circ}$ of tilting motion 

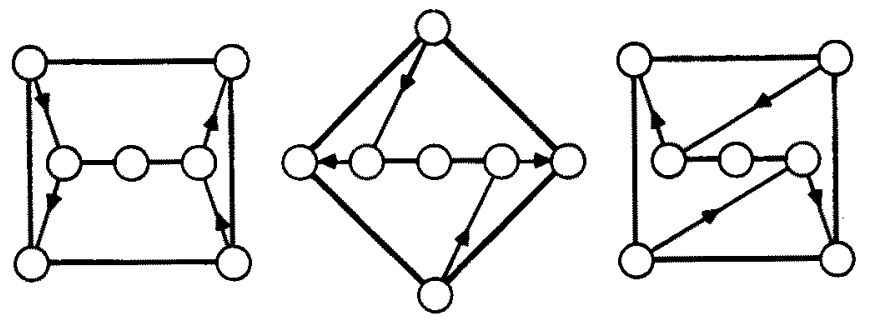

Figure 6. The platform swivels, two of the actuators' contributions to the swiveling torque vanish while the other two's reach their maximum mechanical advantage. A similar situation occurs for all the topological region transitions, which explains the exceptional extent of the working region having high kinematic conditioning.

in both directions and $270^{\circ}$ of swivel. Of course, this ideal performance must be compromised for kinematic performance, structural properties, and space to lodge bearings and actuators. What has actually been achieved in the first prototype is about $90^{\circ}$ of tilting motion in both directions and $180^{\circ}$ of swivel. These figures are expected to be improved substantially in a future prototype.

The large workspace increase makes it possible to take full advantage of the efficiency of parallel linkages as discussed in [9]. In effect, during most motions of the joint all four actuators move, each contributing mechanical power combined at the output link, because the actuator efforts sum and they all move. The worst case occurs when two actuator velocities vanish, in which case these actuators may only play a structural role. The best case occurs when all four actuators move at the same velocity and exert the same effort.

In a competing serial arrangement, during movements where the joint is kinematically well conditioned, only one actuator is producing mechanical power, the others have low velocity and uselessly dissipate power to support their neighbors, while the strength of the joint is limited by the weakest link. The worst case occurs when the joint is near a singularity when some actuators move at high speed exerting little effort, thus operating at low efficiency. Moreover, in a serial arrangement the stiffness of the group is limited by the stiffness of the weakest joint, whereas in the parallel case, their stiffness add.

As a result of the previous observations, excellent strength-to-weight ratio and power-to-weight ratio are expected over a large workspace, made possible by 'actuator redundancy'. The moving mass lumped at the output link is about $1 \mathrm{Kg}$ at a lever arm of about $0.1 \mathrm{~m}$, resulting in a moment of inertia of the order $0.01 \mathrm{Kg} . \mathrm{m}^{2}$. Considering that the joint can produce a torque of $200 \mathrm{~N} . \mathrm{m}$ (with a low supply pressure of $3.3 \mathrm{MPa}-500 \mathrm{psi}$ ), flat within a bandwidth of at least $100 \mathrm{~Hz}$ in isometric conditions around any of the three rotation axes, its performance can be quite remarkable. We are now in position to examine the tradeoffs which were confronted to come up with a practical shoulder joint. 


\subsection{Dimensioning}

The investigations carried out to determine the range of parameters for a useful device resulted in several important observations reported in [13]. It was found that conventional kinematic indices could in fact be quite misleading in the search for a useful device if physical limitations were not taken into account. Another important and related observation is the low sensitivity of kinematic performance as a function of the design parameters, except at scattered trouble spots. This was typified by the need to use log scales to concisely summarize results. The upshot of the study is that kinematic design for manipulators is better described as the avoidance of debilitating conditions rather than targeting for sharp optima.

When $\mathbf{J}$ is the manipulator Jacobian, $\sigma_{1}, \sigma_{2}, \sigma_{3}$ are its singular values, $k(\mathbf{J})=\sigma_{1} / \sigma_{3}$ is its condition number, three such indices were used in a hierarchical design method: global conditioning, actuator forces minimization, and global gradient index. The second and third indices were introduced for the first time:

$$
\begin{aligned}
D_{g}=\frac{\int_{\mathcal{W}} D_{l} d w}{\int_{\mathcal{W}} d w}=\frac{\int_{\mathcal{W}} 1 / k(\mathbf{J}) d w}{\int_{\mathcal{W}} d w} \\
F_{g}=\frac{\int_{\mathcal{W}} F_{l} d w}{\int_{\mathcal{W}} d w}=\frac{\int_{\mathcal{W}} 1 / \sigma_{3} d w}{\int_{\mathcal{W}} d w} \\
G D_{g}=\max _{\mathcal{W}} G D_{l}=\max _{\mathcal{W}}\left\|\nabla D_{l}\right\|
\end{aligned}
$$

Referring to Fig. 3, it was found that the general case mechanism could be made isotropic with a conditioning ideally flat for the values $l_{b}=l_{p}=2 l_{d}$ (units are irrelevant: since it is a spherical mechanism, its properties are scale invariant). In practice, for a shoulder mechanism, there is no reason why it should be isotropic. Actually, it should have greater strength around the axis working against gravity. The scale of the device is directly related to the stroke of the actuators.

Due to the vast number of actual design parameters, a trial and error was used to precisely determine the final dimensions of the device: first with the construction of a series of physical models of increasing fidelity and then checking with the indices listed above that performance was indeed acceptable. Most importantly, the decision tree was rooted in the actuator choice (see section 2.5). At the first level, many decisions were dictated by the necessity of pistontype actuators to have their shortest length exceed their maximal stroke. From this observation, attaching the cylinders by their ends would necessarily lead to poorly conditioned mechanisms. To overcome this difficulty, the cylinders must protrude and be placed in cradles, thus ruling out use the square platform (Fig. 3) as the output link. The final dimensioning decisions where made by considering avoidance of self collisions and structural strength (see section 2.6). The entire decision process resembled searching for a Nash equilibrium (conflicting objectives) in game theoretic optimization problem [20]. 


\subsection{Reduction of Passive Joints Count}

One fundamental disadvantage of parallel mechanisms (particularly spatial mechanisms) is the need for numerous passive joints. In the case studied in this paper, a straightforward implementation of the diagram shown on Fig. 3replacing each spherical joint by a three axis gimbal (each with two forks) and providing for torsion decoupling bearings for linear displacement sensors piggy backed on the pistons-leads to a large number of bearings (45). Unless great care is exercised with respect to the strength and precision of all of these joints, wear, backlash, or failure (not mentioning high cost) is to be expected, defeating the supposed advantages of parallel mechanisms. This problem is further compounded by the lack of room usually available to lodge these bearings.

Inoue et al. [11] designed an iso-static platform based on three pantograph (5-bar) mechanisms which displays advantages over the piston driven McCallion-Truong design [14]. While this design reduces the number of passive joints by having a pair of pistons share two revolute joints in a double Hooke joint arrangement, further saving can be obtained by noticing that a single revolute joint is needed to orient a plane - the plane containing the pantograph. This fact was also noticed by Dunlop et al. [6], who further pointed out that this would save torsion decoupling bearings for linear sensors mounted on screw actuators. The combination of these ideas to the case of piston actuators leads to a simple structure illustrated Fig. 7. A structurally sound implementation of this chain can be realized with 25 bearings, which saves almost half the number of bearings as compared to the straightforward design.

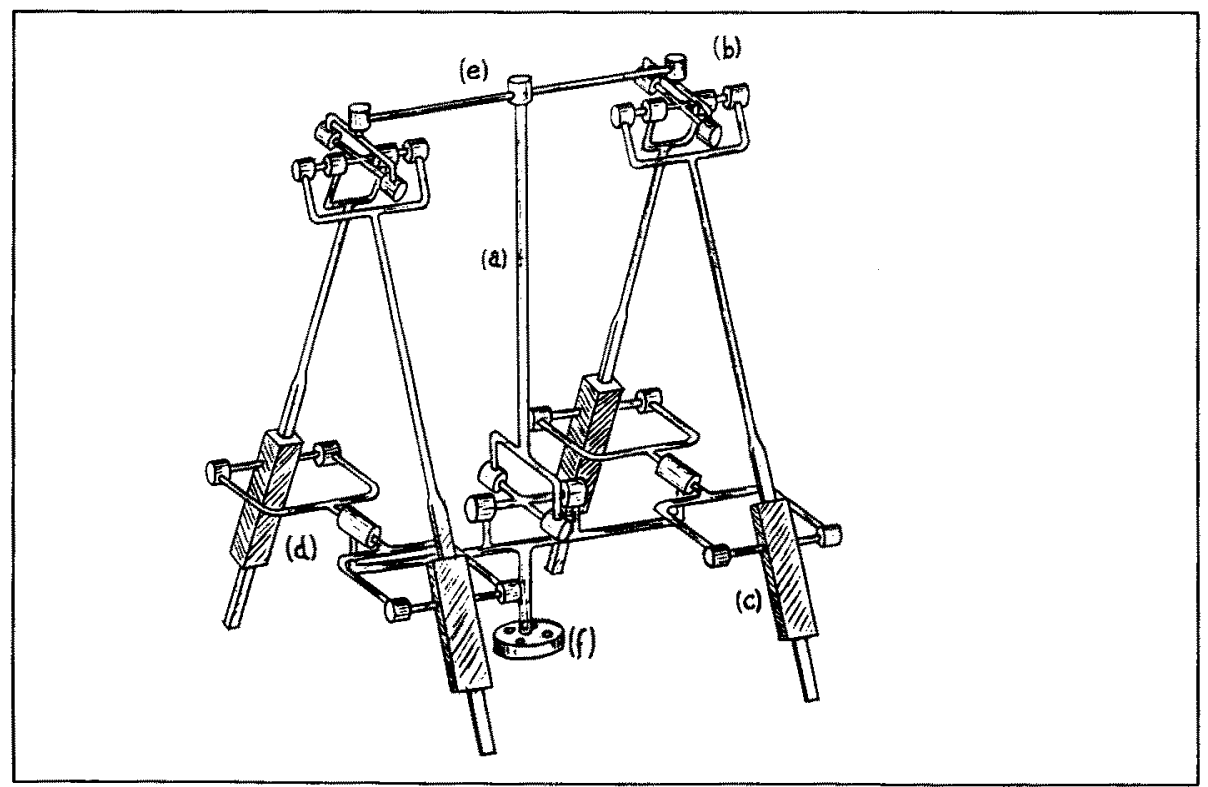

Figure 7. Complete kinematic chain. 
The bearings, although supporting large loads, could be made very small and yet have little backlash thanks to a technique developed at the Center for Engineering Design at the University of Utah.

Finally, yet another benefit of actuator redundancy contributes to ensure the elimination of the residual backlash. In any given orientation of the mechanism, internal stresses can be actively created, independently from the external load, thus bias-loading all the mechanism's bearings.

\subsection{Actuators}

It is suggested here that the actuator analysis problem in robotics might be split into four items:

- Energy Storage. Under this heading, one considers the form under which the energy needed to actuate the robotic device is stored. In a manufacturing application, this question of is small importance since it can reasonably be assumed that an unlimited supply of energy is available. In other applications of robotics, this item can play a major role, for example for planetary explorations vehicles or untethered submarine rovers, in which case a capacious energy storage must be part of the robot itself.

- Energy Transport. One considers the method by which energy is transported from storage to a final stage, where it must be available in mechanical form. In most cases, this function is performed in multiple steps. For example in an electric industrial robot, energy is transported in electrical form, converted into mechanical form in the manipulator's motors and then, most often, once again mechanically transported to the joints via gears, shafts, belts, chains, cables, tendons, hydraulic or pneumatic conduits, or other mechanical energy transmission techniques. A 'direct drive' robot is characterized by the absence of mechanical transmission of energy, except by the structure of the manipulator itself (hence most hydraulic robots are direct drive).

- Energy Throttling. One considers here the method whereby energy is throttled to ensure control over the robot. Energy throttling is hard to achieve mechanically (clutches do that inefficiently). Among a large number of possibilities, electric energy throttling is usually the method of choice, even in a multi-step process as for electromagnetic servovalves. Regardless of the type of energy being throttled, there are three basic methods. In the first method, constant power is delivered by the supply, and the throttling mechanism arranges for a variable amount of the effort to be diverted to the load, usually by creating an effort imbalance. An electric version of this principle leads to 'class $A$ ' amplifiers, a hydraulic version of it leads to the jet pipe hydraulic valve. In the second method, the power delivered by the supply varies with that delivered to the load ('class B') limiting the loss in the throttling mechanism. But in this case the throttling mechanism must have large bandwidth because the 
power must be switched from throttling to quiescence, and vice versa, at each reversal of the direction of the energy flow. In the third method, in some sense a limiting case of the previous case, power is switched, and switching timing is used to throttle power. Then it must have even higher bandwidth to approximate ideal switching. This topic is not often studied in robotics, although it is quite a thorny problem more often than generally assumed (example: variable reluctance electric actuators).

- Energy Conversion. Under this last item, one must examine which principle is applied to convert a transportable form of energy into mechanical energy. The reader is referred to $[10]$ for a recent survey.

Most actuator systems accomplish transport, throttling and conversion in multiple steps. Hydraulic techniques have been selected for use in this shoulder joint prototype enabling direct application in areas in which new manipulator designs are in need, in particular telerobotics. In this area, often, the levels of performance are attainable only with hydraulic actuation. Also the recently available force-controlled ASI integrated hydraulic actuators offered a path toward rapid creation of a high performing prototype. In a number of telerobotic applications, there is need for human-like performance in terms of bulk, precision, strength, reach and dexterity. Again, only hydraulic actuators can be made compact enough to even consider approaching the level human performance. Thus, with this in mind, the four items listed above can be discussed. ${ }^{2}$

Because of the large amount of power required by hydraulic actuators, the energy storage can only be utility-supplied, or be stored chemically in conjunction with a first conversion stage provided by a turbine or an internal combustion engine. This is the method used in many 'pre-robotic' devices such as cranes, excavators, forestry or mining equipment. In submarine applications, high levels of power may be made available through umbilical cables. With hydraulics, the transport function is accomplished with hydraulic lines which must be run from the source of pressure to the actuators. Lines have their own dynamical properties which are actually best described as distributed parameters systems. For this reason, their dynamics are not simple and they can account for significant losses in energy and performance. Hence, compact high-bandwidth valves that can be co-located with the actuators themselves are quite advantageous from a control view point.

With this technique, excellent performance using local compensation can be obtained, even with long and ill-modelled lines. The valve itself is only a part of the energy throttling mechanism since it is driven by an electromagnetic motor, itself driven by an electronic amplifier. In the case of the ASI actuators it was found that the electromagnetic energy conversion mechanism was in fact a limiting factor in the performance of the complete actuator. The last stage of the energy throttling mechanism is based on forcing the fluid through orifices whose areas can be controlled and the last stage of energy conversion is accomplished simply by letting the fluid pressure act differentially on the

\footnotetext{
${ }^{2}$ It a worthwhile to reflect on Nature's solution to these four problems.
} 
sides of pistons or vanes. Details on the properties and compensation control of these actuators can be found in [1].

\subsection{Structural Design and Collision Avoidance}

The structural design was carried out while attempting to take full advantage of the opportunities offered by parallel linkage mechanisms. For example, pistons, as opposed to rotary vane actuators, can be used as structural members and thus economically contribute to strength. Piston actuators have in general numerous structural advantages over rotary actuator while being simpler to manufacture and maintain, and they have greater efficiency.

The principal structural parts are listed by order of decreasing design difficulty: (a) Central stem (1 unit); (b) Double Hooke joints (2 identical units); (c) Actuator cradles (4 identical units); (d) Double actuator forks (2 identical units); (e) Output flange (1 unit); (f) Ground link (1 unit); As it can be seen, the number of parts is quite reasonable are they can be made to have rather simple shapes. The loads types and magnitudes in each bearing was examined, and correspondingly sized.

In the current state of prototype, no systematic structural optimization was carried out, using finite element methods for example, but that could be done in the future. It is encouraging to see that the first sizing estimates used in this first prototype already produced quite a light and compact system. Certain deficiencies were noticed and will have to be corrected in future prototypes.

The main culprit in the reduction of the practical workspace is the possibility of self collisions well within the singularity-free work region. This topic is not studied in any systematic fashion because of the great difficulty in its formalization. General guidelines have been followed to alleviate this problem. Ideally all parts should be as small as possible, but of course, this approach has its limits.

Except for the output flange which is made of aluminum, the material selected for the construction of most of the structure is stainless steel to optimize the strength to size ratio of its parts.

\section{Experimental Results}

The shoulder joint was tested in the laboratory using a L-shaped member designed to approximate the construction of a complete arm. It is made of two steel tubes welded at a right angle with lengths $0.3 \mathrm{~m}$ each. It is bolted to the output flange shoulder such that the axis of first tube coincides with its axis of symmetry. This member is quite rigid and has a mass of $1.6 \mathrm{Kg}$. An additional load mass of $1.4 \mathrm{Kg}$ is affixed to the end of the second tube. The moments of inertia of this composite load about the joint center of rotation are approximately $0.62 \mathrm{Kg} \cdot \mathrm{m}^{2}, 0.48 \mathrm{Kg} \cdot \mathrm{m}^{2}$, and $0.15 \mathrm{Kg} \cdot \mathrm{m}^{2}$.

In an attempt to decouple the basic performance of the design from that of the various control algorithms currently under investigation, the simplest feedback controller is used. It is a proportional position feedback controller about each actuator, augmented by a lag term for improved steady-state accuracy. 
The controller is implemented digitally at a servo rate of $1 \mathrm{kHz}$. We have also implemented a force regulator of similar design.

We executed a small step motion about the axis of highest inertia, in the middle of the joint range. In this condition, the shoulder is capable of producing accelerations of $130 \mathrm{~m} / \mathrm{s}^{2}$ at the tip where the wrist joint is to be located, and a maximum tip velocity of $0.45 \mathrm{~m} / \mathrm{s}$ was observed. The maximum static force produced at the wrist varies from a worst case of $200 \mathrm{~N}$ to a best case of 400 $\mathrm{N}$, according to direction of the exerted force, at the center of the shoulder joint range. The force control bandwidth is observed to be about about $100 \mathrm{~Hz}$. Position resolution could not be measured with the available equipment.

The basic low velocity and force resolution performance is not a function of the design since it can be qualified of being "direct drive" (there are no motion transmission elements other than simple linkages interposed between the actuators and the load). Low velocity performance and force resolution depend completely on the actuators, sensors and local feedback control. To date, experiments in this area have been limited solely by the resolution of the 12 bits analog to digital converters used for digital control.

\section{Conclusion}

A manipulator shoulder joint design based on a combinatorial mechanism which addresses several issues of concern in telerobotics was described. A large workspace was achieved despite the use of parallel linkages, thereby offering an opportunity to take advantage of their possibilities in terms of structural strength and precision. The joint is efficient because any motion in any direction combines the contribution of at least 3 and almost everywhere of 4 actuators, where a serial joint could use only one actuator. They are also advantages from a control view point, in terms of impedance modulation and control robustness, when compared to a serial arrangement as described in [2].

As a result of the preceding items, a dramatic improvement in power-toweight ratio has been achieved while construction was kept simple and the number of parts minimized, while low pressure hydraulics led to a significant torque output. Moreover, four identical piston type actuators, which are the simplest to manufacture and to maintain, were used exclusively.

This design provides a path toward the development of a highly reliable system since it has built-in redundancy. There is natural provision for self calibration: sensors, kinematics, and dynamics. Initial success in this direction has been achieved [16]. Self-testing is natural extension of self calibration: Work is under way to investigate the automatic diagnostic of partial structural, actuator, and sensor failures as well as backlash. Finally, there is built-in provision for degraded modes of operation with missing sensors, missing actuators and minor structural failures. Moreover backlash can be actively compensated.

At the time of this writing, an elbow joint using two actuators identical to those used in the shoulder is under construction. A compact wrist mechanism with four smaller actuators is also in the design stage. All these joints are based on design principles described in this paper and will result in a manipulator 


\section{8}

arm with high dexterity, high strength-to-weight ratio, powered by 6 replicated piston actuators and 4 smaller ones, also identical.

\section{Acknowledgments}

I am indebted to many people who contributed to this project in various ways: in particular Dr. Fraser Smith and David Barret from Sarcos Research Corp. for their first class engineering design and prototype realization, Prof. Stephen C. Jacobsen for his encouragements and valuable comments, Benoit Boulet and Ronald Kurtz, for their excellent work, Prof. Laeeque Daneshmend for his contribution to modeling and control of actuator systems, Chafye Nemri and Duncan Baird for their engineering support. Finally, I would like to thank Profs. I. W. Hunter and J. M. Hollerbach for their support within the project "High-Performance Manipulators" (C3) funded by IRIS, the Institute for Robotics and Intelligent Systems part of Canada's National Centers of Excellence program (NCE). Additional funding was provided by a team grant from FCAR, le Fond pour les Chercheurs et l'Aide à la Recherche, Québec, and an operating grant from NSERC, the National Science and Engineering Council of Canada.

\section{References}

[1] Boulet, B., Daneshmend, L. K., Hayward, V., Nemri, C. 1993. System Identification and Modelling of a High Performance Hydraulic Actuator. Experimental Robotics 2, Chatila, R., Hirzinger, G. (Eds.), Lecture Notes in Control and Information Sciences, Springer Verlag.

[2] Boulet, B., Hayward, V. 1993. Robust control of a robot joint with hydraulic actuator redundancy. Part I: Theory application and controller design. Part II: Practical variations and experimentation. TR-CIM-93-6 and TR-CIM-93-7. Technical Report, McGill Center for Intelligent Machines. McGill University, Montréal Canada.

[3] Burdick, J. W. and Chirikjan. Hyper-redundant robots: kinematics and experiments. in Preprints of 6th International Symposium on Robotics Research, Oct. 2-5, Hidden Valley, PA.

[4] Charentus, S., Renaud, M. 1990. Modelling and Control of a Modular, Redundant Robot Manipulator. in Experimental Robotics 1, Hayward, V., Khatib, O. (Eds.), Lecture Notes in Control and Information Sciences 139. Springer Verlag, pp, 508-527.

[5] Dietrich, J. Hirzinger, G., Gombert, B., Schott, J. 1990. On a unified concept for a new generation of light-weight robots. in Experimental Robotics 1, Hayward, V., Khatib, O. (Eds.), Lecture Notes in Control and Information Sciences 139. Springer Verlag, pp. 244-286.

[6] Dunlop, G. R., Johnson, G. R., Afzulpurkar. 1991. Joint design for extended range Stewart platform applications. Proc. Eight World Congress on the Theory of Machines and Mechanisms. Prague, Czechoslovakia. Vol. 2. pp. 449-451.

[7] Hayward V. 1993. Borrowing some design ideas from biological manipulators to design an artificial one. In Robots and Biological Systems, NATO Series, P. Dario, P. Aebisher, and G. Sandini, (Eds.), Springer Verlag. pp. 139-151. 
[8] Hayward, V. Kurtz, R. 1991. Modeling of a parallel wrist mechanism with actuator redundancy. In Advances in Robot Kinematics. Stifter, S., Lenarcic (eds.). Springer-Verlag. pp. 444-456.

[9] Hirose, S., and Sato, M. 1989. Coupled drive of the multi-DOF robot. in Proc. IEEE Int. Conf. on Robotics and Automation, pp. 1610-1616.

[10] Hollerbach, J. M., Hunter, I. W., Ballantyne, J. 1991. A comparative analysis of actuator technologies for robotics. In Robotics Review 2, Khatib, O., Craig, J., Lozano-Perez, T. (eds.), MIT Press.

[11] Inoue, H., Tsukasa, Y., Fukuizumi, T. 1986. Parallel manipulator. In The Third International Symposium on Robotics Research. Faugeras, O. E., Giralt, G. (Eds.). MIT Press. pp. 321-327.

[12] Jacobsen, S. C., Smith, F. M., Backman, D. K., and Iversen, E. K. 1991. High performance, high dexterity, force reflective teleoperator II. in Proc. ANS Topical Meeting on Robotics and Remote Systems.

[13] Kurtz, R., Hayward, V. 1992. Multi-goal optimization of a parallel mechanism with actuator redundancy. IEEE Transactions on Robotics and Automation. Vol. 8, No. 5. pp 644-651.

[14] McCallion, H., Truong, P. D. 1979. The analysis of a six degree of freedom work station for mechanized assembly. Proc. 5th World Congress on the Theory of Machines and Mechanisms, pp 611-616.

[15] Morecki, A., Malczyk, G. 1987. Mathematical Model of a flexible manipulator of the elephant's-trunk-type. in Proc. Sixth CISM-IFToMM Symposium on Theory and Practice of Robot and Manipulators, Morecki, A., Bianchi, G., Kedzior, A. (Eds.), Hermes, Paris.

[16] Navhi, A., Hollerbach, J. M., Hayward, V. Calibration of a parallel robot using multiple kinematic loops. Submitted to 1994 IEEE Int. Conf. on Robotics and Automation.

[17] Pierrot, F., Dauchez, P., Fournier, A. 1991. Hexa a fast six-dof fully parallel robot. Proc. 5th Int. Conf. on Advanced Robotics, Pisa, Italy. IEEE Press.

[18] Rosheim, M. E., 1989. Robot wrist actuators, John Wiley \& Sons.

[19] Regan, B. 1991. ATLAS-8F Advanced Bilateral Manipulator System. In Proc. of ROV-91 Conference, Marine Technology Society. Washington DC.

[20] Vincent, T. L. 1983. Game theory as a design tool. J. of Mechanisms, Transmissions, and Automation in Design, Trans. of ASME, Vol. 105, No. 1, pp. 165-170.

[21] Yoshinada, Hiroshi, Yamazaki, T., Suwa, T., Naruse, T. 1992. Design and control of a manipulator system driven by seawater hydraulic actuator. Proc. Second Int. Symposium on Measurement and Control in Robotics (ISMCR), Tsukuba Science City, Japan, pp. 359-364. 